\title{
Schematic model for QCD. III. Hadronic states
}

\author{
M. V. Nuñez, S. H. Lerma, and P. O. Hess \\ Instituto Ciencias Nucleares, Universidad Nacional Autónoma de México, Apdo. Postal 70-543, México 04510 Distrito Federal \\ S. Jesgarz \\ Instituto de Fisica, Universidad de São Paulo, CP 66318, São Paulo, 05315-970, São Paulo, Brasil \\ O. Civitarese and M. Reboiro \\ Departamento de Fisica, Universidad Nacional de La Plata, c.c. 67 1900, La Plata, Argentina.
}

(Received 24 February 2004; published 28 September 2004)

\begin{abstract}
The hadronic spectrum obtained in the framework of a QCD-inspired schematic model is presented. The model is the extension of a previous version, whose basic degrees of freedom are constituent quarks, antiquarks, and gluons. The interaction between quarks and gluons is a phenomenological interaction and its parameters are fixed from data. The classification of the states, in terms of quark and antiquark and gluon configurations is based on symmetry considerations, and it is independent of the chosen interaction. Following this procedure, nucleon and $\Delta$ resonances are identified, as well as various penta- and hepta-quarks states. The lowest pentaquarks state is predicted at $1.5 \mathrm{GeV}$ and it has negative parity, while the lowest hepta-quarks state has positive parity and its energy is of the order of $2.5 \mathrm{GeV}$.
\end{abstract}

DOI: 10.1103/PhysRevC.70.035208

PACS number(s): 12.90.+b, 21.90. $+\mathrm{f}$

\section{INTRODUCTION}

In a series of previous publications [1-3], we have proposed a schematic model to describe some features of the nonperturbative regime of QCD. The main ingredients of the model can be found in Ref. [1], where we have presented some results on the meson spectrum of QCD. In Ref. [2] we have studied the formation of fermion and boson condensates. A preliminary set of results, about the energy and parity of systems of the type $q^{3 n}(q \bar{q})^{m}$, was presented in Ref. [3].

As it was discussed in Ref. [1], the model describes, qualitatively, the main features of the meson spectrum of QCD. This is an encouraging result because it shows that, even in the conditions of low energy QCD, e.g., large coupling constants and nonconservation of particle number, the use of schematic models may be very useful to explore hadronic properties. The model of Ref. [1] belongs to the class of algebraic models [4] which describe the interaction between fermions and bosons. Some other examples, of fermion-boson Hamiltonians, can be found in Refs. [5-7]. The work of Ref. [8] gives the classification of the spectrum of many gluon states, by applying symmetry principles. In Ref. [8], the predictions of the model concerning states with exotic quantum numbers were tested.

The purpose of this work is to extend the model of Ref. [1] to describe, qualitatively, the main structure of the hadronic spectrum of QCD. The essentials of the model are the following:

(a) color, flavor, and spin degrees of freedom are taken explicitly into account to construct the configurations, both in the quark and gluon sectors of the model;

(b) the orbital part of the quark and antiquark wave functions is represented by $s$ waves; and

(c) the interaction of the quarks with the gluons takes place via gluon pairs coupled to spin and color zero. Other possible gluon states are considered as spectators.

The present work is organized as follows. Section II gives a brief description of the model. We shall avoid as much as possible the repetition of details which can be found in the already published papers [1-3], and focus on the aspects which are relevant for the description of the hadronic spectrum. In Sec. III we discuss the classification of many quarkantiquark states, and the classification of many gluon states [8]. In Sec. IV we show and discuss the hadronic spectrum predicted by the model, as a natural step following the study presented in Ref. [1], where the meson sector was analyzed. Conclusions are drawn in Sec. V.

\section{THE MODEL}

The basic degrees of freedom of the model are represented by the level scheme shown in Fig. 1. The quarks occupy two levels $\left( \pm \omega_{f}\right)$, of negative and positive energies. Antiquarks are depicted as holes in the lower level. If the operators $c_{\alpha i}^{\dagger}$ and $c^{\alpha i}$ create and annihilate quarks in the upper $(i=1)$ and lower $(i=2)$ level, the relation to quark (antiquark) creation and annihilation operators is given by

$$
\begin{gathered}
\mathbf{a}_{\alpha}^{\dagger}=\mathbf{c}_{\alpha 1}^{\dagger}, \quad \mathbf{d}_{\alpha}=\mathbf{c}_{\alpha 2}^{\dagger} \\
\mathbf{a}^{\alpha}=\mathbf{c}^{\alpha 1}, \quad \mathbf{d}^{\dagger \alpha}=\mathbf{c}^{\alpha 2},
\end{gathered}
$$

where the index $\alpha$ refers to color, flavor and spin quantum numbers. Since three degrees of color, three degrees of flavor (only $u, d$, and $s$ quarks are taken into account) and two degrees of spin are considered, each level is 18 -fold degenerate. The energy of each level is one-third of the nucleon mass and it represents the constituent (effective) mass of the quarks. In this picture, quarks are confined to a sphere of radius $R=1 \mathrm{fm}$ thus the mass originates in the kinetic energy. 


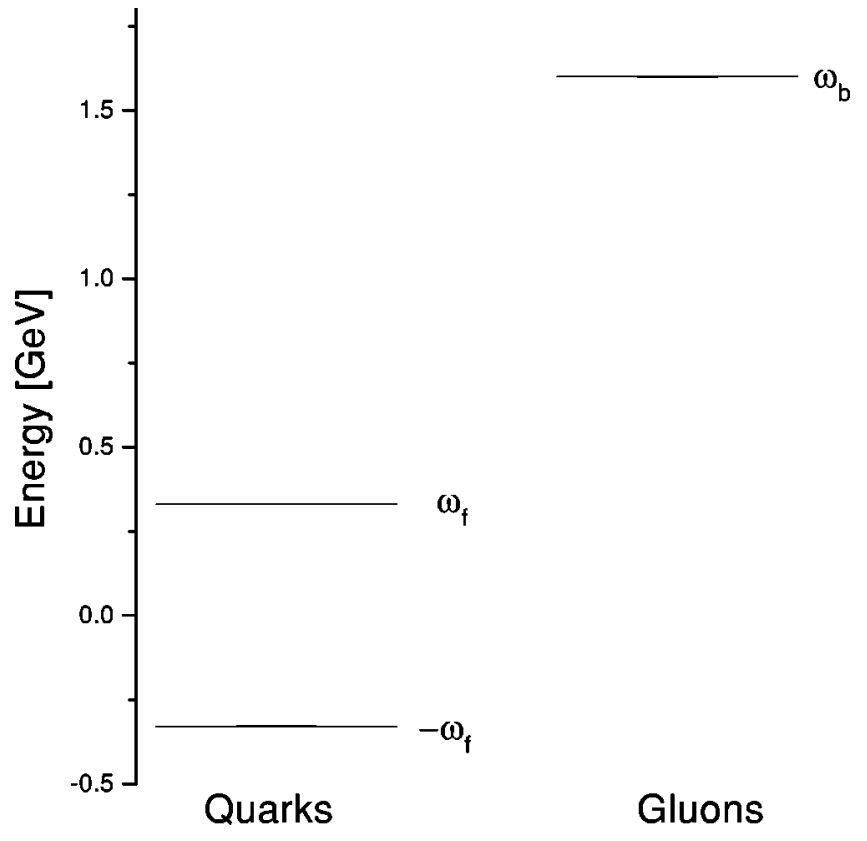

FIG. 1. Schematic representation of the model space. The fermion levels are indicated by their energies $\pm \omega_{f}$. The gluon-pairs are represented by the level at the energy $\omega_{b}$.

The model describes quarks and antiquarks moving in orbital $s$ states. Orbital excitations can be accounted for by increasing the number of degrees of freedom.

To this system of quarks we add a boson, which represents pairs of gluons of color and spin zero. Its energy, $\omega_{b}$, is fixed as it was done in Ref. [8]. The coupling between quarks and antiquarks and gluons includes only these gluon-pair configurations. The couplings to other pairs of gluons are neglected because their contributions to the meson spectrum are not important [1].

The basic excitations in the fermion sector are $q \bar{q}$ pairs, which are described by $B_{\lambda f, S M}^{\dagger}$ and $B^{\lambda f, S M}$ pair operators [1], where $\lambda=0,1$ stands for flavor states $(0,0)$ and $(1,1)$, and the spin $S$ can be 0 or 1 . These pair of fermions, a subclass of bifermion operators $[6,7]$, can be mapped onto a boson space [9]. We denote the corresponding operators by $b_{\lambda f, S M}^{\dagger}$.

This is the basic structure of the model presented previously [1]. In order to extend it to the description of baryons, and because we work in the boson space of quark-antiquark pairs, one should add three valence quarks via the operators $\left[D^{\left(\lambda_{0}, \mu_{0}\right) S_{0}} \times a^{\dagger}\right]_{f, m}^{(\lambda, \mu)}$, where two ideal quark operators $a_{\alpha}^{\dagger}[9]$ are coupled as a diquark $D_{f_{0} m_{0}}^{\left(\lambda_{0}, \mu_{0}\right) S_{0}}=\left[a^{\dagger} \times a^{\dagger}\right]_{f_{0} m_{0}}^{\left(\lambda_{0}, \mu_{0}\right) S_{0}}$ with flavor, spin $\left(\lambda_{0}, \mu_{0}\right) S_{0}$ either $(0,1) 0$ or $(2,0) 1$.

The model Hamiltonian is written as in Ref. [1], with the inclusion of new terms which are needed for the description of baryons, namely

$$
\begin{aligned}
\boldsymbol{H}= & 2 \omega_{f} \boldsymbol{n}_{f}+\omega_{b} \boldsymbol{n}_{b}+\sum_{\lambda S} V_{\lambda S}\left\{\left[\left(\boldsymbol{b}_{\lambda S}^{\dagger}\right)^{2}+2 \boldsymbol{b}_{\lambda S}^{\dagger} \boldsymbol{b}_{\lambda S}+\left(\boldsymbol{b}_{\lambda S}\right)^{2}\right]\right. \\
& \left.\times\left(1-\frac{\boldsymbol{n}_{f}}{2 \Omega}\right) b+\boldsymbol{b}^{\dagger}\left(1-\frac{\boldsymbol{n}_{f}}{2 \Omega}\right)\left[\left(\boldsymbol{b}_{\lambda S}^{\dagger}\right)^{2}+2 \boldsymbol{b}_{\lambda S}^{\dagger} \boldsymbol{b}_{\lambda S}+\left(\boldsymbol{b}_{\lambda S}\right)^{2}\right]\right\} \\
& +\boldsymbol{n}_{(0,1) 0}\left[D_{1} \boldsymbol{n}_{b}+D_{2}\left(\boldsymbol{b}^{\dagger}+\boldsymbol{b}\right)\right]+\boldsymbol{n}_{(2,0) 1}\left[E_{1} \boldsymbol{n}_{b}+E_{2}\left(\boldsymbol{b}^{\dagger}+\boldsymbol{b}\right)\right],
\end{aligned}
$$

where $\left(\boldsymbol{b}_{\lambda S}^{\dagger}\right)^{2}=\left(\boldsymbol{b}_{\lambda S}^{\dagger} \cdot \boldsymbol{b}_{\lambda S}^{\dagger}\right)$ is a short hand notation for the scalar product. ${ }^{1}$ The factor $\left(1-\boldsymbol{n}_{f} / 2 \Omega\right)$ simulates the effect of the terms which would appear in the exact boson mapping of the quark-antiquark pairs [9,7]. The operators $b^{\dagger}$ and $b$ are boson creation and annihilation operators of the gluon pairs with spin and color zero.

The interaction describes scattering and vacuum fluctuation terms of fermion and gluon pairs. The strength $V_{\lambda S}$ is the same for each allowed value of $\lambda$ and $S$, due to symmetry reasons, as shown in Ref. [1]. These parameters were determined in Ref. [1] and they are taken as fixed values in the present calculations. The matrix elements of the meson part of the Hamiltonian are calculated in a seniority basis. For details, please see Ref. [1]. The operators $\boldsymbol{n}_{\left(\lambda_{0}, \mu_{0}\right) S_{0}}$ are number operators of the diquarks coupled to flavor-spin $\left(\lambda_{0}, \mu_{0}\right) S_{0}$. The interaction does not contain terms which connect states with different hypercharge and isospin. It does not contain flavor mixing terms, either. Therefore, in the comparison with data, the energies should be corrected by the mass formula given in Ref. [10], with parameters deduced as in Ref. [11] and turning off flavor mixing terms. The procedure is explained in details in Ref. [1].

The last two terms of Eq. (2) are needed to describe the interaction between valence quarks and meson-like states. The parameters $\omega_{f}$ and $\omega_{b}$ were fixed at 0.33 and $1.6 \mathrm{GeV}$, respectively. The parameters $V_{\lambda S}$ were adjusted to the mesonic spectrum and their values are: $V_{00}=0.0337 \mathrm{GeV}, V_{01}$ $=0.0422 \mathrm{GeV}, V_{10}=0.1573 \mathrm{GeV}$, and $V_{11}=0.0177 \mathrm{GeV}$, respectively. This shows the strong effects of the $V_{10}$ interaction, which acts on pairs coupled to flavor $(1,1)$ and spin 0 (pion-like states). The parameters $D_{k}$ and $E_{k}(k=1,2)$ are adjusted to the nucleon resonances and $\Delta$ resonances, respectively. Their values are $D_{1}=-1.442 \mathrm{GeV}, D_{2}=-0.439 \mathrm{GeV}$, $E_{1}=-1.187 \mathrm{GeV}$, and $E_{2}=-0.362 \mathrm{GeV}$.

\section{THE MODEL SPACE}

The complete classification of the many quark-antiquark system was given in Ref. [1]. Here we will summarize it and discuss the aspects which are relevant for the present calculations. The main idea about the classification of the many quark-antiquark system is to treat quarks in the two level system of Fig. 1. The ground state (vacuum), for the unperturbed situation (zero interaction), is given by 18 quarks occupying the lowest level. An $n$ particle-hole excitation of this ground state represents $n$ physical quarks and antiquarks. Therefore, baryons are represented by three physical quarks in the upper level. Particles with a structure of the form $q^{3}(q \bar{q})^{n}$ are described by three valence quarks plus $n$ particlehole excitations. The group chain, describing these states, is given by

\footnotetext{
${ }^{1}$ Similarly for $\left(b_{\lambda S}\right)^{2}$ and $\left(b_{\lambda S}^{\dagger} b_{\lambda S}\right)$.
} 


$$
\begin{array}{ccc}
{\left[1^{N}\right]} & {[h]=\left[h_{1} h_{2} h_{3}\right]} & {\left[h^{T}\right]} \\
U(4 \Omega) & \supset U\left(\frac{\Omega}{3}\right) \otimes & U(12) \\
& \cup & \cup \\
\left(\lambda_{C}, \mu_{C}\right) S U_{C}(3) & {\left[v_{1} v_{2} v_{3} v_{4}\right]} & U(4) \otimes S U_{f}(3)\left(\lambda_{f}, \mu_{f}\right) \\
& & \cup \\
& & S U_{S}(2) S, M,
\end{array}
$$

where the irreducible representation (irrep) of $U(4 \Omega)$ is the completely antisymmetric one and $N$ is the number of particles involved. $\Omega=9$ is the degeneracy corresponding to 3 degrees of freedom in the color sector and three degrees of freedom in flavor [1]. Similar group chains can be constructed for larger values of $\Omega$. In Eq. (3), $\left[h^{T}\right]$ is the transposed Young diagram of $[h][13,16]$. Due to the antisymmetric irrep $\left[1^{N}\right]$ of $U(4 \Omega)$ the irreps of $U(\Omega / 3)$ and $U(12)$ are complementary and the irrep of $U(\Omega / 3)$ has maximally three rows [13]. In the group chain (3) no multiplicity labels are indicated. There is a multiplicity $\rho_{f}$ for $\left(\lambda_{f}, \mu_{f}\right)$ and $\rho_{S}$ for the spin $S$. The color labels $\left(\lambda_{C}, \mu_{C}\right)$ are related to the $h_{i}$ via $\lambda_{C}=h_{1}-h_{2}$ and $\mu_{C}=h_{2}-h_{3}$. The irrep of the $U(4)$ group is given by a Young diagram with four rows, i.e., $\left[v_{1}, v_{2}, v_{3}, v_{4}\right]$. The complete state is given by

$$
\left|N,\left[v_{1} v_{2} v_{3} v_{4}\right]\left(\lambda_{C}, \mu_{C}\right), \rho_{f}\left(\lambda_{f}, \mu_{f}\right) Y T T_{z}, \rho_{S} S M\right\rangle,
$$

where $Y$ is the hypercharge, $T$ is the isospin and $T_{z}$ its third component. For meson-like states, the color quantum numbers to be considered are $\left(\lambda_{C}, \mu_{C}\right)=(0,0)$.

To obtain the values of $h_{i}$ one has to consider all possible partitions of $N=h_{1}+h_{2}+h_{3}$. For colorless states we have $h_{1}$ $=h_{2}=h_{3}=h$. Each partition of $N$ appears only once. The irrep [hhh] of $U(\Omega / 3)=U(3)(\Omega=9)$ fixes the irrep of $U(12)$, as indicated in Eq. (3). For $\Omega=9$ and color zero the meson irrep of $U(12)$ is given by $\left[3^{6} 0^{6}\right]$ and for baryons it is $\left[3^{7} 0^{5}\right]$.

In Table I we list the partial $U(4) \times U_{f}(3)$ content for meson states of the $U(12)$ irrep $\left[3^{6} 0^{6}\right]$ and in Table II the partial content of the $U(4)$ irreps listed in Table I. The irreps which are shown contain up to two quark-antiquarks pairs only [12]. The $U(4)$ irreps have in general four rows while the $U_{f}(3)$ of the flavor group have three rows $\left[p_{1} p_{2} p_{3}\right]$. The adopted notation for flavor is $(\lambda, \mu)$ where $\lambda=p_{1}-p_{2}$ and $\mu$ $=p_{2}-p_{3}$. The minimal number of quarks in an $U(4)$ irrep is given by the sum of the last two rows of the $U(4)$ Young diagram while the number of antiquarks is given by the difference between $2 \Omega=18$ and the sum of the first two rows of the $U(4)$ Young diagram. In the spin representation for quarks $\left(\left[q_{1} q_{2}\right]\right)$, the sum $n_{q}=q_{1}+q_{2}$ gives the number of quarks and $S_{q}=\left(q_{1}-q_{2}\right) / 2$ is the spin carried by the quarks. Similarly, for antiquarks $\left(\left[\overline{q_{1}} \overline{q_{2}}\right]\right) S_{\bar{q}}=\left(\overline{q_{1}}-\overline{q_{2}}\right) / 2$ and $2 \Omega$ $-n_{q}=\overline{q_{1}}+\overline{q_{2}}$. This is related to the fact that the unperturbed ground state, of the two level model space, contains 18 quarks, as it was defined before. The total spin $S$ of the system can be obtained via the condition $\left|S_{q}-S_{q}\right| \leqslant S \leqslant S_{q}$ $+S_{\bar{q}}$. In Tables III and IV the classification of some of the baryon states is given. In Table III only $U(4) \otimes U_{f}(3)$ states
TABLE I. Partial $U(4) \times U_{f}(3)$ content of the $U(12)$ irrep $\left[3^{6} 0^{6}\right]$ (color zero, meson-like states). The flavor irreps $(0,0),(1,1),(3,0)$, $(0,3),(2,2)$ describe the flavor singlet, octet, decuplet, antidecuplet, and 27-plet, respectively. The multiplicity of the $U(4) \times U_{f}(3)$ content in the $U(1)$ irrep $\left[3^{6} 0^{6}\right]$ is given in the last column.

\begin{tabular}{lccc}
\hline \hline$U(4)$ & $U_{f}(3)$ & $(\lambda, \mu)$ & Mult \\
\hline$[8811]$ & {$[666]$} & $(0,0)$ & 1 \\
{$[8811]$} & {$[765]$} & $(1,1)$ & 1 \\
{$[8811]$} & {$[864]$} & $(2,2)$ & 1 \\
{$[9711]$} & {$[765]$} & $(1,1)$ & 1 \\
{$[9711]$} & {$[774]$} & $(0,3)$ & 1 \\
{$[9711]$} & {$[855]$} & $(3,0)$ & 1 \\
{$[8820]$} & {$[765]$} & $(1,1)$ & 1 \\
{$[8820]$} & {$[774]$} & $(0,3)$ & 1 \\
{$[8820]$} & {$[855]$} & $(3,0)$ & 1 \\
{$[9720]$} & {$[666]$} & $(0,0)$ & 1 \\
{$[9720]$} & {$[765]$} & $(1,1)$ & 1 \\
{$[9720]$} & {$[864]$} & $(2,2)$ & 1 \\
{$[9810]$} & {$[765]$} & $(1,1)$ & 1 \\
{$[9900]$} & {$[666]$} & $(0,0)$ & 1 \\
\hline \hline
\end{tabular}

are considered which contain five quarks and two antiquarks. In Table IV only the lowest states which contain unusual flavor are listed. The $U(12)$ irrep is given by $\left[3^{7} 0^{5}\right]$.

To extend the space of meson-like configurations [1], we shall include the flavor irreps $(3,0),(0,3)$, and $(2,2)$, since all of these contain at least two quark-antiquark pairs. The irreps $(3,0)$ and $(0,3)$ have total spin 1 while the irrep $(2,2)$ may have total spin values 0,1 , and 2 . Within our boson representation of the quark-antiquark pairs, the $(3,0)$ and $(0,3)$ irreps with spin 1 can be obtained via the coupling scheme [e.g., for $(3,0)]\left[b_{(1,1) 0}^{\dagger} \times b_{(1,1) 1}^{\dagger}\right]_{f_{0} m=1}^{(3,0) 1}|0\rangle$ while the irrep $(2,2) S=0,1,2$ comes from $\left[b_{(1,1) 1}^{\dagger} \times b_{(1,1) 1}^{\dagger}\right]_{f_{0} m=S}^{(2,2) S=0,1,2}|0\rangle$, $\left[b_{(1,1) 0}^{\dagger} \times b_{(1,1) 0}^{\dagger}\right]_{f_{0} m=S}^{(2,2) S=0}|0\rangle$. The two configurations with $S=0$

TABLE II. Quark-antiquark content of some $U(4)$ meson-like irreps. The table shows the irreps which contain at most two quarks and two antiquarks.

\begin{tabular}{lccccccc}
\hline \hline$U(4)$ & {$\left[q_{1} q_{2}\right]$} & $n_{q}$ & $S_{q}$ & {$\left[\overline{q_{1}} \overline{q_{2}}\right]$} & $n_{\bar{q}}$ & $S_{\bar{q}}$ & $S$ \\
\hline$[8811]$ & {$[11]$} & 2 & 0 & {$[88]$} & 2 & 0 & 0 \\
{$[9711]$} & {$[11]$} & 2 & 0 & {$[97]$} & 2 & 1 & 1 \\
{$[8820]$} & {$[20]$} & 2 & 1 & {$[88]$} & 2 & 0 & 1 \\
{$[9720]$} & {$[20]$} & 2 & 1 & {$[97]$} & 2 & 1 & $0,1,2$ \\
{$[9810]$} & {$[10]$} & 1 & $\frac{1}{2}$ & {$[98]$} & 1 & $\frac{1}{2}$ & 0,1 \\
{$[9810]$} & {$[11]$} & 2 & 0 & {$[97]$} & 2 & 1 & 1 \\
{$[9810]$} & {$[11]$} & 2 & 0 & {$[88]$} & 2 & 0 & 0 \\
{$[9810]$} & {$[20]$} & 2 & 1 & {$[97]$} & 2 & 1 & $0,1,2$ \\
{$[9810]$} & {$[20]$} & 2 & 1 & {$[88]$} & 2 & 0 & 1 \\
{$[9900]$} & {$[00]$} & 0 & 0 & {$[99]$} & 0 & 0 & 0 \\
{$[9900]$} & {$[10]$} & 1 & $\frac{1}{2}$ & {$[98]$} & 1 & $\frac{1}{2}$ & 0,1 \\
{$[9900]$} & {$[20]$} & 2 & 1 & {$[97]$} & 2 & 1 & $0,1,2$ \\
\hline \hline
\end{tabular}


TABLE III. Partial $U(4) \times U_{f}(3)$ content of the $U(12)$ irrep $\left[3^{7} 0^{5}\right]$ (color zero, baryon-like states). The flavor irreps $(0,0),(1,1)$, $(3,0),(0,3),(2,2),(4,1)$ describe the flavor singlet, octet, decuplet, anti-decuplet, 27-plet, and 35-plet, respectively. The multiplicity of the $U(4) \times U_{f}(3)$ content of the $U(1)$ irrep $\left[3^{7} 0^{5}\right]$ is given in the last column. The first three rows give some higher lying $U(4)$ irreps which are associated with a flavor irrep $(3,3)$.

\begin{tabular}{|c|c|c|c|}
\hline$U(4)$ & $U_{f}(3)$ & $(\lambda, \mu)$ & Mult \\
\hline [8832] & [1074] & $(3,3)$ & 1 \\
\hline [8841] & [1074] & $(3,3)$ & 1 \\
\hline [9732] & [1074] & $(3,3)$ & 1 \\
\hline [9822] & [777] & $(0,0)$ & 1 \\
\hline [9822] & {$[876]$} & $(1,1)$ & 1 \\
\hline [9822] & [966] & $(3,0)$ & 1 \\
\hline [9822] & [975] & $(1,1)$ & 1 \\
\hline [9831] & [777] & $(0,0)$ & 1 \\
\hline [9831] & {$[876]$} & $(1,1)$ & 2 \\
\hline [9831] & [966] & $(3,0)$ & 1 \\
\hline [9831] & {$[885]$} & $(0,3)$ & 1 \\
\hline [9831] & {$[975]$} & $(2,2)$ & 1 \\
\hline [9831] & [1065] & $(4,1)$ & 1 \\
\hline [8850] & [777] & $(0,0)$ & 1 \\
\hline [8850] & {$[876]$} & $(1,1)$ & 1 \\
\hline [8850] & {$[885]$} & $(0,3)$ & 1 \\
\hline [8850] & [975] & $(2,2)$ & 1 \\
\hline [9840] & {$[876]$} & $(1,1)$ & 1 \\
\hline [9840] & [975] & $(2,2)$ & 1 \\
\hline [9821] & {$[876]$} & $(1,1)$ & 1 \\
\hline [9930] & [966] & $(3,0)$ & 1 \\
\hline
\end{tabular}

are linear combinations of the $U(4)$ irreps [9720] and [8811]. For $S=1$ the coupling scheme is $\left[b_{(1,1) 0}^{\dagger} \times b_{(1,1) 1}^{\dagger}\right]_{f_{0} m=S}^{(2,2) S=1}|0\rangle$, where $f_{0}$ denotes the flavor state with maximum weight.

In the boson representation the states are given by the direct product of the eigenstates of one-, three-, eight-, and 24-dimensional harmonic oscillators [1]. For each harmonic oscillator the basis states are given by

$$
\mathcal{N}_{N_{\lambda S} \nu_{\lambda S}}\left(\boldsymbol{b}_{\lambda S}^{\dagger} \cdot \boldsymbol{b}_{\lambda S}^{\dagger}\right)^{\frac{N_{\lambda S^{-}} \nu_{\lambda S}}{2}}\left|\nu_{\lambda S} \alpha_{\lambda S}\right\rangle
$$

where $N_{\lambda S}$ is the number of bosons of type $[\lambda, S], \nu_{\lambda S}$ is the seniority, and $\mathcal{N}_{N_{\lambda S}{ }^{\nu} \lambda S}$ is a normalization factor. The seniority is the number of uncoupled $\boldsymbol{b}_{\lambda S}$ bosons. The quantity $\alpha_{\lambda S}$ contains all other quantum numbers needed to specify a particular harmonic oscillator. The state (5) is the superposition of a certain number of quark-antiquark pairs coupled to a given flavor spin. This number of quark-antiquark pairs will be used to denote nontrivial configurations.

The Hamiltonian changes only the number of boson pairs of the type $\left(b_{\lambda S}^{\dagger} \cdot b_{\lambda S}^{\dagger}\right)$, and it leaves the seniority invariant. The number of these boson pairs is not conserved. Thus, we can divide the Hilbert space in seniority subspaces. Each subspace includes all combinations of quark-antiquark pairs of a given flavor spin. The spectrum obtained for a fixed
TABLE IV. Quark-antiquark content of some $U(4)$ baryon irreps. These irreps contain the nucleon and $\Delta$ resonances (last two rows) and penta- and heptaquark-states with unusual flavor. The first three rows shows the content of the irreps which are associated with flavor irreps $(3,3)$ (see first three rows in Table III). The double line separates high lying irreps from low lying ones.

\begin{tabular}{lccccccc}
\hline \hline$U(4)$ & {$\left[q_{1} q_{2}\right]$} & $n_{q}$ & $S_{q}$ & {$\left[\overline{q_{1}} \overline{q_{2}}\right]$} & $n_{q}^{-}$ & $S_{\bar{q}}$ & $S$ \\
\hline$[8832]$ & {$[32]$} & 5 & $\frac{1}{2}$ & {$[88]$} & 2 & 0 & $\frac{1}{2}$ \\
{$[8841]$} & {$[41]$} & 5 & $\frac{3}{2}$ & {$[88]$} & 2 & 0 & $\frac{3}{2}$ \\
{$[9732]$} & {$[32]$} & 5 & $\frac{1}{2}$ & {$[97]$} & 2 & 1 & $\frac{1}{2}, \frac{3}{2}$ \\
{$[9831]$} & {$[41]$} & 5 & $\frac{3}{2}$ & {$[88]$} & 2 & 0 & $\frac{3}{2}$ \\
{$[9831]$} & {$[32]$} & 5 & $\frac{1}{2}$ & {$[88]$} & 2 & 0 & $\frac{1}{2}$ \\
{$[9831]$} & {$[32]$} & 5 & $\frac{1}{2}$ & {$[97]$} & 2 & 1 & $\frac{1}{2}, \frac{3}{2}$ \\
{$[8850]$} & {$[50]$} & 5 & $\frac{5}{2}$ & {$[88]$} & 2 & 0 & $\frac{5}{2}$ \\
{$[9840]$} & {$[50]$} & 5 & $\frac{5}{2}$ & {$[88]$} & 2 & 0 & $\frac{5}{2}$ \\
{$[9840]$} & {$[50]$} & 5 & $\frac{5}{2}$ & {$[97]$} & 2 & 1 & $\frac{3}{2}, \frac{5}{2}, \frac{7}{2}$ \\
{$[9840]$} & {$[41]$} & 5 & $\frac{3}{2}$ & {$[97]$} & 2 & 1 & $\frac{1}{2}, \frac{3}{2}, \frac{5}{2}$ \\
{$[9831]$} & {$[31]$} & 4 & 1 & {$[98]$} & 1 & $\frac{1}{2}$ & $\frac{1}{2}, \frac{3}{2}$ \\
{$[9840]$} & {$[40]$} & 4 & 2 & {$[98]$} & 1 & $\frac{1}{2}$ & $\frac{3}{2}, \frac{5}{2}$ \\
{$[9921]$} & {$[21]$} & 3 & $\frac{1}{2}$ & {$[0]$} & 0 & 0 & $\frac{3}{2}$ \\
{$[9930]$} & {$[30]$} & 3 & $\frac{3}{2}$ & {$[0]$} & 0 & 0 & $\frac{3}{2}$ \\
\hline \hline
\end{tabular}

flavor spin and seniority combinations will be identical to another one corresponding to a different flavor spin but with the same seniority. This leads to the appearance of a degeneration in the spectrum.

A similar classification can be given for the baryonic sector. Within the boson representation, the ideal valence quarks are added to describe baryons. One has to couple the mesonlike states with the states $(1,1) 1 / 2$ or with $(3,0) 3 / 2$, to which the three valence quarks can be coupled, to describe nucleon and $\Delta$ resonances, respectively. This implies a degeneration of different flavor-spin irreps. As an example, if the configuration $(1,1) 1 / 2^{+}$of the three valence quarks is coupled to a meson irrep $(1,1) 0^{-}$then the resulting allowed flavor-spin irreps are $(0,0) 1 / 2^{-},(1,1) 1 / 2^{-},(3,0) 1 / 2^{-}$, $(0,3) 1 / 2^{-}$, and $(2,2) 1 / 2^{-}$.

Another ingredient of the model is the nonconservation of the particle number (quarks, antiquarks and gluons). This is a fundamental property of a relativistic theory with large interaction constants. It means that physical particles, in general, do not contain a fixed number of quarks, antiquarks and/or gluons but rather an average number of them. In this picture, the nucleons will not contain, mainly, three valence quarks but also an average number of quark-antiquark and gluon pairs. The mixing of particle number is needed to remove the multiplicity of states at low energy, a feature which any constituent quark model exhibits when the number of quarks, antiquarks and gluons is fixed.

We summarize, for completeness, the configurations which may appear in the gluon sector of the theory. In the model of Ref. [8] the basic building blocks are effective gluons with color $(1,1)$ and spin 1 . In Table $\mathrm{V}$ we show all states of glue-balls with color zero and maximally six effective gluons. The lowest glue-ball state is the first excited 
TABLE V. The classification of many-gluon states, with color $(0,0)$. The multiplicity of the configuration $(0,0)$ is one up to six gluons. For the notation of the groups, which appear in the manygluons states, and their irreps see Ref. [8], where the general classification, i.e., with open color, can be deduced. $P$ and $C$ refer to the parity and charge conjugation of the gluon states, respectively.

\begin{tabular}{|c|c|c|c|c|}
\hline$U(8)(U(3))$ & $O(8)$ & $S O(3)(J)$ & $P$ & $C$ \\
\hline$[2]$ & $(0000)$ & 0,2 & +1 & +1 \\
\hline$[4]$ & $(0000)$ & $0,2,4$ & +1 & +1 \\
\hline$\left[2^{2}\right]$ & $(0000)$ & 0,2 & +1 & +1 \\
\hline$[6]$ & $(0000)$ & $0,2,4,6$ & +1 & +1 \\
\hline$[42]$ & $(0000)$ & $0,2_{2}, 3,4$ & +1 & +1 \\
\hline$\left[2^{3}\right]$ & $(0000)$ & 0 & +1 & +1 \\
\hline$[3]$ & $(3000)$ & 1,3 & -1 & -1 \\
\hline$[5]$ & $(3000)$ & $1,3,5$ & -1 & -1 \\
\hline$[41]$ & $(3000)$ & $1,2,3,4$ & -1 & -1 \\
\hline$[32]$ & $(3000)$ & $1,2,3$ & -1 & -1 \\
\hline$\left[1^{3}\right]$ & (1110) & 0 & -1 & +1 \\
\hline$\left[31^{2}\right]$ & (1110) & 0,2 & -1 & +1 \\
\hline$\left[21^{2}\right]$ & $(2110)$ & 1 & +1 & -1 \\
\hline$\left[41^{2}\right]$ & (2110) & 1,3 & +1 & -1 \\
\hline$[321]$ & (2110) & 1,2 & +1 & -1 \\
\hline$\left[2^{2}\right]$ & $(2200)$ & 0,2 & +1 & +1 \\
\hline$[42]$ & $(2200)$ & $0,2_{2}, 3,4$ & +1 & +1 \\
\hline$[321]$ & (2200) & 1,2 & +1 & +1 \\
\hline$\left[2^{3}\right]$ & $(2200)$ & 0 & +1 & +1 \\
\hline$\left[2^{2} 1\right]$ & (2210) & 1 & -1 & -1 \\
\hline$\left[31^{2}\right]$ & $(3110)$ & 0,2 & -1 & -1 \\
\hline$[6]$ & $(6000)$ & $0,2,4,6$ & +1 & +1 \\
\hline$[42]$ & $(4200)$ & $0,2_{2}, 3,4$ & +1 & +1 \\
\hline$\left[41^{2}\right]$ & $(4110)$ & 1,3 & +1 & -1 \\
\hline$\left[3^{2}\right]$ & $(3300)$ & 1,3 & +1 & -1 \\
\hline$\left[2^{3}\right]$ & $(2220)$ & 0 & +1 & +1 \\
\hline
\end{tabular}

$J^{\pi C}=0^{++}$state, where $J$ is the angular momentum, $\pi$ is the parity and $C$ is the charge conjugation. For details, see Ref. [8].

\section{APPLICATIONS}

In this section we shall show and discuss the results of the model introduced in the previous section, concerning baryon states. Since the Hamiltonian conserves flavor, spin, and parity, all states belonging to the same flavor irrep are degenerate. In order to introduce a splitting in the isospin and hypercharge the Gel'man-Okubo interaction [14] should be added. Otherwise, the parameters of the Hamiltonian should be adjusted to reproduce the corrected experimental masses. This is the procedure which we have adopted in the calculations. Next Sec. IV A is devoted to the results concerning mesonic states, others than the ones reported in Ref. [1], like those with flavor $(2,2)$. These states illustrate the role of some additional degeneracies of the model, and their possible physi- cal implications. In Sec. IV B we present the results for nucleon resonances, as well as some results about more exotic states, like the predicted energy and parity of the lowest penta- and hepta-quarks states. Finally, in Sec. IV C we analyze the case of $\Delta$ resonances. The experimental data are taken from Refs. [15].

\section{A. Meson states}

In Table VI we list the quark-antiquark and gluon contents of some selected mesonic states. The quantity $n_{\lambda S}$ is the average number of $q \bar{q}$ pairs coupled to flavor $(\lambda, \mu)$ and spin $S$. The quantity $n_{g}$ is the average number of gluon pairs coupled to color singlet and spin zero. The seniority content is given by the value $(v)=\left(v_{00}, v_{01}, v_{10}, v_{11}\right)$, where $v_{\lambda S}$ refers to the seniority in the channel $(\lambda, \lambda) S$. Some of the states shown in Table VI belongs to flavor irreps which contain exotic combinations of hypercharge and isospin not allowed in the simplest $q \bar{q}$ system. From the seniority content one sees that these states contain at least two $q \bar{q}$ pairs.

While the states with flavor $(3,0)$ and $(0,3)$ have energies of the order of $1.5 \mathrm{GeV}$, the state with flavor $(2,2)$ lies at very low energy, nearly at the same energy of the $f_{0}(980)$ state. Experimentally, the $f_{0}$ state lies at approximately twice the energy of the $\eta(541)$ state. Their dominant decay into $K^{+} K^{-}$leads to the interpretation of these states as $K^{+} K^{-}$-molecules. Within our model the $(2,2) 0^{+}$state is built upon the seniority $\nu=2$ state $\left[b_{(1,1) 0}^{\dagger} \times b_{(1,1) 0}^{\dagger}\right]_{f, 0}^{(2,2) 0}|0\rangle$. The highest weight state is given by $b_{(1,1) f_{0}, 0}^{\dagger} b_{(1,1) f_{0}, 0}^{\dagger}|0\rangle$, which is the direct product of two pairs of the type $(1,1) 0^{-}$. This can be interpreted, within the present model, as a configuration of two $q \bar{q}$-like mesons. The difference between the mass of the two pairs and the mass of the $(2,2) 0^{+}$state is about $0.4 \mathrm{GeV}$, which is larger than the observed value. One has to keep in mind that the present model is rather schematic and that it does not include a flavor mixing interaction which could mix the $(1,1) 0^{-}$states with $(2,2) 0^{-}$states, and may induce a coupling of the $f_{0}$ state to molecular states. The model predicts also states of the type $(q \bar{q})^{2}$ which can be coupled to flavor irreps $(3,0)$ and $(0,3)$ (see Tables I and II). The distribution of seniorities is (0011) and the energy of the states is of the order of $1.44 \mathrm{GeV}$. The irreps $(3,0)$ and $(0,3)$ contain isospin-hypercharge configurations which cannot be reached by a single quark-antiquark pair.

\section{B. Nuclear resonances, penta- and hepta-quarks}

In Table VII we show the results for some selected nucleon resonances, as they appear in the schematic model. The content of $q \bar{q}$ pairs of the type $(0,0) 0^{-}$and $(0,0) 1^{-}$are not listed because they are small for the considered cases. Since the Hamiltonian does not change spin, parity, color, and flavor, all states belonging to the same flavor irrep are degenerate. In order to remove the degeneracy one may still add the Gel'man-Okubo term plus a term describing the flavor dependence. We follow the prescription of Ref. [10] with the parameters given in Ref. [11].

The quark, antiquark, and gluon contents of the nucleon are given in the first row of Table VII. The number of $q \bar{q}$ 
TABLE VI. Particle content for selected meson states. In the columns we indicate the theoretical energy, $E_{\text {theo }}$, the flavor, $\left(\lambda_{f}, \mu_{f}\right)$, spin, $J$, parity, $\pi$, seniority content, $(v)=\left(v_{00} v_{01} v_{10} v_{11}\right)$, the expectation value of the boson pairs in the channels $(0,0) 0^{-}\left(n_{00}\right),(0,0) 1^{-}\left(n_{01}\right),(1,1) 0^{-}\left(n_{10}\right)$, and $(1,1) 1^{-}\left(n_{11}\right)$ and the total number of gluon pairs $\left(n_{g}\right)$ with spin 0 . In the last three rows some states, not reported in Ref. [1], are listed. They contain exotic combinations of hypercharge and isospin. The experimental data are taken from Ref. [15]. The $X(797)$ is interpreted as a molecular state. Note that, for the particles in the first $(0,0),(1,1) 0^{-}$and $(0,0),(1,1) 1^{-}$irreps, we are listing the value of the mass without flavor mixing; they are marked by an asterisk (see also Ref. [1] for further explanation).

\begin{tabular}{lccccccccc}
\hline \hline Particle & $E_{\text {theo }}$ & $\left(\lambda_{f}, \mu_{f}\right)$ & $J^{\pi}$ & $(v)$ & $n_{00}$ & $n_{01}$ & $n_{10}$ & $n_{11}$ & $n_{g}$ \\
\hline Vacuum & 0.0 & $(0,0)$ & $0^{+}$ & $(0000)$ & 0.00 & 0.03 & 3.11 & 0.03 & 1.705 \\
$f_{0}(400-1200)$ & 0.656 & $(0,0)$ & $0^{+}$ & $(0000)$ & 0.00 & 0.01 & 0.46 & 0.01 & 0.32 \\
$f_{0}(980)$ & 0.797 & $(1,1)$ & $0^{+}$ & $(0020)$ & 0.00 & 0.02 & 3.78 & 0.02 & 1.49 \\
$f_{1}(1420)$ & 1.445 & $(0,0)$ & $1^{+}$ & $(0011)$ & 0.00 & 0.02 & 2.39 & 1.02 & 0.90 \\
$f_{2}(1270)$ & 1.363 & $(1,1)$ & $1^{+}$ & $(0110)$ & 0.00 & 1.03 & 2.46 & 0.02 & 0.99 \\
$\eta^{\prime}(958)^{*}$ & 0.885 & $(0,0)$ & $0^{-}$ & $(1000)$ & 1.01 & 0.02 & 2.51 & 0.02 & 1.29 \\
$\eta(1440)$ & 1.379 & $(0,0)$ & $0^{-}$ & $(1000)$ & 1.00 & 0.01 & 0.77 & 0.01 & 0.44 \\
$\eta(541)^{*}$ & 0.602 & $(1,1)$ & $0^{-}$ & $(0010)$ & 0.00 & 0.02 & 2.71 & 0.03 & 1.16 \\
$\eta(1295)^{*}$ & 1.428 & $(1,1)$ & $0^{-}$ & $(0010)$ & 0.00 & 0.01 & 1.61 & 0.01 & 0.53 \\
$\eta(1760)$ & 1.671 & $(1,1)$ & $0^{-}$ & $(1020)$ & 1.01 & 0.02 & 3.53 & 0.02 & 1.25 \\
$\omega(782)^{*}$ & 0.851 & $(0,0)$ & $1^{-}$ & $(0100)$ & 0.04 & 1.03 & 2.56 & 0.02 & 1.34 \\
$\phi(1020)^{*}$ & 0.943 & $(1,1)$ & $1^{-}$ & $(0001)$ & 0.00 & 0.02 & 2.39 & 1.02 & 1.19 \\
$\omega(1420)$ & 1.389 & $(1,1)$ & $1^{-}$ & $(0001)$ & 0.00 & 0.01 & 0.85 & 1.01 & 0.47 \\
$\omega(1600)$ & 1.639 & $(1,1)$ & $1^{-}$ & $(0120)$ & 0.00 & 1.03 & 3.55 & 0.02 & 1.28 \\
$X(1440)$ & 1.440 & $(3,0)$ & $1^{+}$ & $(0011)$ & 0.00 & 0.02 & 2.39 & 1.02 & 0.90 \\
$X(1440)$ & 1.440 & $(0,3)$ & $1^{+}$ & $(0011)$ & 0.00 & 0.02 & 2.39 & 1.02 & 0.90 \\
$X(797)$ & 0.797 & $(2,2)$ & $0^{+}$ & $(0020)$ & 0.00 & 0.02 & 3.78 & 0.02 & 1.49 \\
\hline \hline
\end{tabular}

pairs is about 0.5 , i.e., including the three valence quarks there are in average 3.5 quarks and 0.5 antiquarks in the nucleon. The number of gluon pairs is approximately 1.4 , i.e., in average there are nearly three gluons present. This implies a $59 \%$ quark content and a $41 \%$ gluon content, a result which is expected from previous evidences. The theoretical Roper resonance approaches the experimental energy of $1.44 \mathrm{GeV}$. This is a nice result, which may be difficult to obtain with other models, except for the constituent quark model of Ref. [17]. In average, there are two $q \bar{q}$ pairs and 1.9

TABLE VII. Particle content for selected baryon states. In columns we indicate the theoretical energy, $E_{\text {theo, }}$, the flavor, $\left(\lambda_{m}, \mu_{m}\right)$, spin, $J_{m}$, and parity, $\pi$, for the meson part; the final flavor irrep, $(\lambda, \mu)$, and spin, $J$, and parity; the seniority content, $(v)=\left(v_{00} v_{01} v_{10} v_{11}\right)$, the expectation value of the boson pairs in the channels $(1,1) 0^{-}\left(n_{10}\right)$ and $(1,1) 1^{-}\left(n_{11}\right)$, and the total number of gluon pairs $\left(n_{g}\right)$ coupled to spin 0 . In the last four rows some additional particles are listed which contain unusual combinations of hypercharge and isospin. The $\Theta^{+}(1540)$ is the reported pentaquarks state. Another pentaquarks state, according to our notation, is called $X(1510)$ while $H_{k}(k=1,2)$ are the lowest heptaquarks states of unusual flavor.

\begin{tabular}{lcccccccc}
\hline \hline Particle & $E_{\text {theo }}$ & $\left(\lambda_{m}, \mu_{m}\right)$ & $J_{m}^{\pi}$ & $(\lambda, \mu) J^{\pi}$ & $(v)$ & $n_{10}$ & $n_{11}$ & $n_{g}$ \\
\hline Nucleon & 0.950 & $(0,0)$ & $0^{+}$ & $(1,1) \frac{1}{2}^{+}$ & $(0000)$ & 0.47 & 0.05 & 1.40 \\
Roper & 1.49 & $(0,0)$ & $0^{+}$ & $(1,1) \frac{1}{2}^{+}$ & $(0000)$ & 1.91 & 0.04 & 1.92 \\
$\mathrm{~N}(1650)$ & 1.51 & $(1,1)$ & $0^{-}$ & $(1,1) \frac{1}{2}^{-}$ & $(0010)$ & 3.14 & 0.03 & 2.81 \\
$\mathrm{~N}(1535)$ & 1.79 & $(1,1)$ & $1^{-}$ & $(1,1) \frac{1}{2}^{-}, \frac{3}{2}^{-}$ & $(0001)$ & 0.38 & 1.00 & 1.04 \\
$\Theta^{+}(1540)$ & 1.51 & $(1,1)$ & $0^{-}$ & $(0,3) \frac{1}{2}^{-}$ & $(0010)$ & 3.14 & 0.03 & 2.81 \\
$X(1510)$ & 1.51 & $(1,1)$ & $0^{-}$ & $(2,2) \frac{1}{2}^{-}$ & $(0010)$ & 3.14 & 0.03 & 2.81 \\
$H_{1}(2451)$ & 2.45 & $(1,1)$ & $1^{+}$ & $(0,0),(1,1)$ & $(0110)$ & 2.87 & 0.03 & 2.27 \\
& & & & $(3,0),(0,3)$ & & & & \\
$H_{2}(2529)$ & 2.53 & $(2,2)$ & $0^{+}$ & $(4,1), \frac{1}{2}^{+}$ & $(0020)$ & 2.73 & 0.03 & 1.24 \\
\hline \hline
\end{tabular}


TABLE VIII. Particle content for selected $\Delta$ states. In columns we indicate the theoretical energy, $E_{\text {theo, }}$, the flavor, $\left(\lambda_{m}, \mu_{m}\right)$, spin, $J_{m}$, and parity, $\pi$, for the meson part. The other quantities are the final flavor irrep, $(\lambda, \mu)$, the spin, $J$, parity, $\pi$, the seniority content, $(v)=\left(v_{00} v_{01} v_{10} v_{11}\right)$, the expectation value of the boson pairs in the channels $(1,1) 0^{-}\left(n_{10}\right)$ and $(1,1) 1^{-}\left(n_{11}\right)$ and the total number of gluon pairs $\left(n_{g}\right)$ with spin 0 . Particles $(X$ and $H)$ with unusual combinations of hypercharge and isospin are listed in the last two rows. The state in the last row can be interpreted as a heptaquarks state. The *, on some states, indicates that the particular state is obtained via a combination with three valence quarks coupled to $(1,1) \frac{1}{2}^{+}$.

\begin{tabular}{lcccccccc}
\hline \hline Particle & $E_{\text {theo }}$ & $\left(\lambda_{m}, \mu_{m}\right)$ & $J_{m}^{\pi}$ & $(\lambda, \mu) J^{\pi}$ & $(v)$ & $n_{10}$ & $n_{11}$ & $n_{g}$ \\
\hline$\Delta(1232)$ & 1.248 & $(0,0)$ & $0^{+}$ & $(3,0) \frac{3}{2}^{+}$ & $(0000)$ & 0.33 & 0.03 & 0.67 \\
$\Delta(1600)$ & 1.57 & $(0,0)$ & $0^{+}$ & $(3,0) \frac{3}{2}^{+}$ & $(0000)$ & 1.93 & 0.04 & 1.60 \\
$\Delta(1620)^{*}$ & 1.51 & $(1,1)$ & $0^{-}$ & $(3,0) \frac{1}{2}^{-}$ & $(0010)$ & 3.14 & 0.03 & 2.81 \\
$\Delta(1700)^{*}$ & 1.79 & $(1,1)$ & $1^{-}$ & $(3,0), \frac{1}{2}^{-}, \frac{3}{2}^{-}$ & $(0001)$ & 0.38 & 1.00 & 1.04 \\
$\Delta(1750)^{*}$ & 2.49 & $(1,1)$ & $0^{+}$ & $(3,0) \frac{1}{2}^{+}$ & $(1010)$ & 2.85 & 0.03 & 2.19 \\
$\Delta(1900)^{*}$ & 2.09 & $(1,1)$ & $0^{-}$ & $(3,0) \frac{1}{2}^{-}$ & $(0010)$ & 1.40 & 0.02 & 0.68 \\
$X(1640)$ & 1.64 & $(1,1)$ & $0^{-}$ & $(4,1),(2,2), \frac{3}{2}^{-}$ & $(0010)$ & 3.00 & 0.03 & 2.18 \\
$H(2530)$ & 2.53 & $(2,2)$ & $0^{+}$ & $(4,1) \frac{3}{2}^{+}$ & $(0020)$ & 2.67 & 0.02 & 0.97 \\
\hline \hline
\end{tabular}

gluon pairs. Thus, the Roper resonance, in the present model, contains an average number of 5 quarks, 2 antiquarks $\left[q^{3}(q \bar{q})^{2}\right]$ and 3.8 gluons. This implies a $65 \%$ content of quarks and a $35 \%$ content of gluons. The quark-gluon content of the Roper resonance differs from that of the nucleon. It has more particles, a fact which is reflected in its larger collective nature and low energy. The first negative parity resonance appears at about $1.5 \mathrm{GeV}$, corresponding to the configuration $(1,1) \frac{1}{2}^{-}$, and the next one at $1.79 \mathrm{GeV}$ shows a degeneracy between the configurations with $J^{\pi}=\frac{1}{2}^{-}$and $\frac{3}{2}^{-}$. The state at $1.51 \mathrm{GeV}$ contains a large amount of $q \bar{q}$ pairs of the type $(1,1) 0^{-}$while the states at $1.79 \mathrm{GeV}$ are nearly pure $q \overline{q g}{ }^{2}$ configurations. These states are good candidates to represent hybrid baryons. In Table VII, states with flavor $(0,3)$ and $(2,2)$ are also listed. These have the same meson content. They are coupled with the three ideal valence quarks, leading to unusual combinations of hypercharge and isospin. These configurations may be associated to pentaquarks states, like $\Theta^{+}(1540)$ [18], whose position have been predicted in Ref. [19]. Other states of the pentaquarks type may exist, which have in common the same quantum numbers in hypercharge and isospin as other nucleon resonances, and, therefore, they may be difficult to identify experimentally. The interpretation of these states as pentaquarks is based on the value of the seniority of the $q \bar{q}$ pair of the type $(1,1) 0^{-}(\nu=1)$ to which the three ideal valence quarks have to be added. In Ref. [3] we have presented a compilation of results about pentaquarks states, as they are predicted by our model. Within the model, the lowest pentaquarks has negative parity in accordance with QCD sum rules and lattice gauge calculations [20-23]. If the orbital spin $L$ is included, pentaquarks states with positive parity may exist with $L=1$. However, these states include an orbital excitation and should appear at higher energies.

The model contains also heptaquarks, which are characterized by two $q \bar{q}$ pairs added to the three ideal valence quarks. The lowest one with unusual flavor, which cannot be described by a plain three-quark system, is at about $2.5 \mathrm{GeV}$
$\left[H_{1}(2451)\right]$ (see Table VII). It has a content of $2.87 q \bar{q}$ pairs of the type $(1,1) 0^{-}\left(n_{10}\right)$ coupled with three ideal valence quarks to the unusual flavor irrep of the type $(3,0)$ with spinparity $\frac{1}{2}^{+}$. This implies a quark content of $78 \%$ and a gluon content of $22 \%$. There are several other heptaquarks states near the same energy, or at slightly higher energies, which contain one $q \bar{q}$ pair of the type $(1,1) 0^{-}$and one of the type $(1,1) 1^{-}$. Also their gluon content is higher by about one extra gluon. Within our model, the parity of the heptaquarks state is positive. Because we are working in a boson space, we should be careful about the appearance of unphysical states [9]. For example, the three valence quarks can be coupled with the meson background with flavor $(2,2)$, e.g., to the flavor irreps $(3,3),(4,1)$, and $(1,4)$. However, only the $(4,1)$, irrep appears in the list of allowed states related to low lying $U(4)$ irreps (see Tables III and IV). The others are, therefore, unphysical. The $(3,3)$ irreps belong to higher configurations of $U(4)$, with $S=\frac{1}{2}$ and $\frac{3}{2}$. This shows the importance of the complete classification, because only a comparison of the states in the boson space with the list of irreps in the fermion space gives the possible allowed states.

\section{C. $\Delta$ resonances}

In a similar manner, as it was discussed in the section of nucleon resonances, we can treat $\Delta$ resonances. In Table VIII we list the results of our calculations.

The simplest form to obtain a $\Delta$ resonance is to couple a diquarks with $(3,0) \frac{3}{2}$ with the meson-like configuration $(3,0) J$. In general, more than one value of $J$ is allowed, depending on the meson state. Coupling $(3,0) \frac{3}{2}$ with $(0,0) 0^{+}$ leads to the $\Delta(1232)$ resonance. The quark-antiquark and gluon content of the $\Delta(1232)$ is somewhat lower than that of the nucleon. The structure of the theoretical state at $1.57 \mathrm{GeV}$ can be compared to the Roper resonance in the nucleon channel. The first negative parity state with flavor $(3,0)$ and spin $\frac{3}{2}$ is predicted at the energy $1.79 \mathrm{GeV}$, and it contains nearly one $q \bar{q}$ pair with flavor-spin $(1,1) 1^{-}$. 


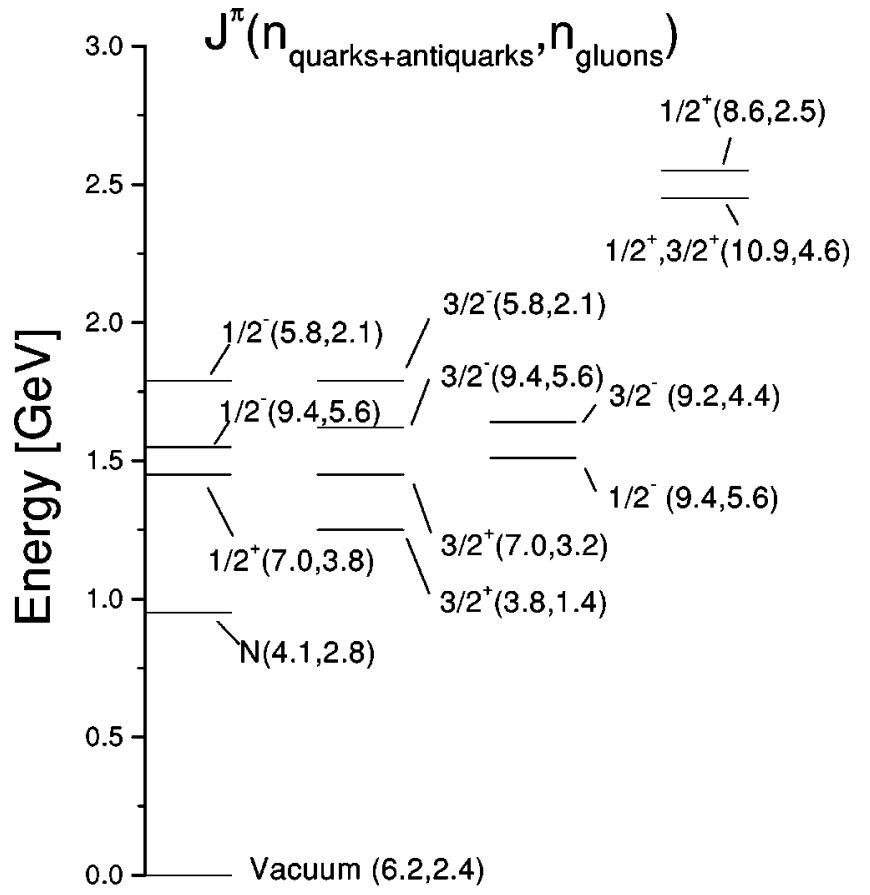

Theoretical Spectrum

FIG. 2. Nucleon resonances (first group of levels), $\Delta$ resonances (second group), pentaquarks (third group), and heptaquarks (fourth group). At the right side of each level are given the assigned spin and parity $\left(J^{\pi}\right)$, and the total quark and antiquark $\left(n_{q}+n_{\bar{q}}\right)$ and gluon $\left(n_{g}\right)$ contents (see the text) The list is not complete. Only some selected states, of physical interest, are listed.

To conclude with this section, we show in Fig. 2 a compilation of the previously reported results.

\section{CONCLUSIONS}

We have extended the schematic model of Ref. [1] to the description of meson states, nucleon resonances, and $\Delta$ reso- nances. The schematic model presented in this work does not conserve the number of quarks, antiquarks, and gluons. Since we are dealing with a model for strong interactions, only the average number of these particles can be extracted from the calculations. It is found that the nucleon has nearly $50 \%$ of gluon content, as expected from previous evidences. The degeneracy of states of the type $q^{n_{1}} q^{n_{2}} g^{n_{3}}$ is removed (see Ref. [1]), but a degeneracy related with the seniority still remains. The Hilbert space is divided into subspaces labeled by the seniority of the different $q \bar{q}$ pairs involved. Subspaces with the same seniority but different flavor and spin are degenerate. This property leads to the prediction of unusual flavorspin combinations which cannot be obtained by working with just one $q \bar{q}$ pair or three quarks. We gave a complete classification for the quarks, antiquarks, and gluons states. The quarks interact with pairs of gluons coupled to spin zero. Other types of gluons are present as spectators [8]. The results of the model are in good agreement with data. The lowest pentaquarks state is predicted at $1.51 \mathrm{GeV}$ [3]. It has an unusual combination of flavor $(0,3) \frac{1}{2}^{-}$and $(2,2) \frac{1}{2}^{-}$, and it has negative parity. Low lying heptaquarks states are predicted, with positive parity, at energies of the order of $2.5 \mathrm{GeV}$. Similar conclusions were reached from the analysis of the results of calculations of $\Delta$ resonances.

As said before, these are features which cannot be obtained by working with three quarks or with one quarkantiquark pair, only. We think that the present results support the notion that the degrees of freedom included in the model may indeed be adequate to describe the low-energy spectrum of QCD. The present conclusions are limited, naturally, by the schematic nature of the proposed Hamiltonian.

\section{ACKNOWLEDGMENTS}

This work belongs to the DGAPA Project No. IN119002. It was partially supported by the National Research Councils of Mexico (CONACYT) and Argentina (CONICET), and by the German Academic Interchange Service of Germany (DAAD).
[1] S. Lerma, S. Jesgarz, P. O. Hess, O. Civitarese, and M. Reboiro, Phys. Rev. C 67, 055209 (2003).

[2] S. Jesgarz, S. Lerma, P. O. Hess, O. Civitarese, and M. Reboiro, Phys. Rev. C 67, 055210 (2003).

[3] N. Nuñez, S. Lerma, S. Jesgarz, P. Hess, O. Civitarese, and M. Reboiro, Phys. Rev. C 70, 025201 (2004).

[4] H. J. Lipkin, N. Meschkov, and S. Glick, Nucl. Phys. A62, 118 (1965).

[5] D. Schütte and J. Da Providencia, Nucl. Phys. A282, 518 (1977).

[6] H. B. Geyer, C. A. Engelbrecht, and F. J. W. Hahne, Phys. Rev. C 33, 1041 (1986).

[7] O. Civitarese and M. Reboiro, Phys. Rev. C 57, 3055 (1998), 58, 2787 (1998),

[8] P. O. Hess, S. Lerma, J. C. López, C. R. Stephens, and A. Weber, Eur. Phys. J. C 9, 121 (1999).
[9] A. Klein and E. R. Marshalek, Rev. Mod. Phys. 63, 375 (1991).

[10] F. Gürsey and L. A. Radicati, Phys. Rev. Lett. 13, 173 (1964).

[11] R. Bijker, M. M. Giannini, and E. Santopinto, UNAM preprint (2003).

[12] S. Lerma, PhD thesis, FC-UNAM, México (2003).

[13] M. Hamermesh, Group Theory and its Application to Physical Problems (Dover, New York, 1989).

[14] W. Greiner and B. Müller, Quantum Mechanics; Symmetries (Springer, Heidelberg, 1989).

[15] Particle Data Group, K. Hagiwara et al., Phys. Rev. D 66, 010001 (2002)

[16] Fl. Stancu, Group Theory in Subnuclear Physics (Oxford University Press, Oxford, 1996).

[17] R. Bijker, F. Iachello, and A. Leviatan, Ann. Phys. (N.Y.) 236, 69 (1994). 
[18] T. Nakano et al., Phys. Rev. Lett. 91, 012002 (2003); DIANA collaboration, V. V. Barmin et al., preprint hep-ex/0304040, Yad. Fiz. (in press); CLAS collaboration, S. Stepanyan et al., Phys. Rev. Lett. 91, 252001 (2003); SAPHIR collaboration, J. Barth et al., preprint hep-ex/307083, Nucl. Phys. (in press); A. E. Asratyan, A. G. Dolgolenko, and M. A. Kubantsev, preprint hep-ex/0309042, submitted to Yad. Fiz.
[19] D. Diakonov, V. Petrov, and M. Polyakov, Z. Phys. A 359, 305 (1997).

[20] Shin-Lin Zhu, Phys. Rev. Lett. 91, 232002 (2003).

[21] J. Sugiyama, T. Doi, and M. Oka, preprint hep-ph/0309271.

[22] F. Csikor, Z. Fodor, S. D. Katz, and T. G. Kovács, e-print hep-lat/0309090

[23] S. Sasaki, e-print hep-lat/0310014. 